\title{
Obese women partially compensate for sucrose added to the diet, without weight gain, over 28 days
}

\author{
R. Hammersley ${ }^{1}$, M. Reid ${ }^{2}$, C. Ballantyne ${ }^{1}$ and M. Duffy ${ }^{3}$ \\ ${ }^{1}$ School of Health and Life Sciences, Glasgow Caledonian University G4 OBA, ${ }^{2}$ Department of Psychology, University of \\ Hull HU6 7RX and ${ }^{3}$ Department of Nutrition, University of Ulster, Coleraine BT52 ISA, UK
}

Sugars, including sucrose, added to the diet in liquid form may displace other nutrients from the diet ${ }^{(1)}$ and may be inadequately compensated for and lead to weight gain ${ }^{(2)}$, although the literature is beset with methodological issues ${ }^{(3)}$. However, some studies have shown that in normal and overweight women, who do not know that they are receiving sucrose because the experimental design is blind, compensate for added sucrose over four weeks by reducing voluntary energy intake, with reductions in voluntary carbohydrate and fat intake $^{(4,5)}$. Aim: To extend this line of experimental research to obese women, who may compensate less accurately. In a between-subjects single blind design, 41 healthy obese (BMI 30-35) women (age 20-50), not currently dieting received sucrose drinks $(4 \times 250 \mathrm{ml}$ total1800 kJ per day; $n=20)$ added to the diet over 4 weeks with equivalent aspartame sweetened drinks for the control group $(n=21)$. As in previous work, women were free-living and food intake was recorded with a detailed, unweighed, prospective food diary used successfully in previous studies ${ }^{(4,5)}$.

By 4 weeks, sucrose supplements significantly reduced voluntary sugar and starch intake from baseline compared to aspartame. Daily energy intake did not increase significantly, nor was there any significant change in body mass index.

It is concluded that when sucrose is given blind obese women partially compensate for the additional energy by reducing voluntary carbohydrate intake, with no signs of adverse effects. These findings are inconsistent with theories which propose that sugars directly increase appetite leading to weight gain. One can infer that among obese women inadequate compensation for sucrose in everyday life may be due primarily to cognitive factors, such as expectations and abstinence violation effects, rather than to adverse physiological effects of sucrose itself. Further research is required to investigate more clearly the psychological and physiological mechanisms underlying appetite regulation in this group. The precise mechanisms remain to be clarified.
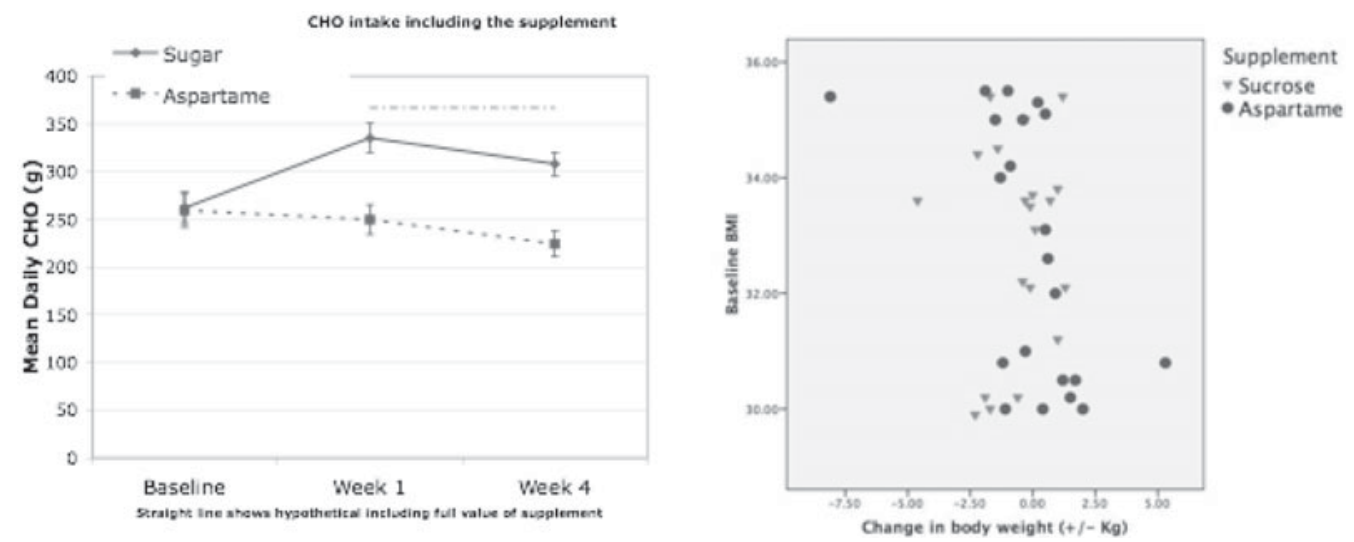

1. Keller KL, Kirzner J, Pietrobelli A, St-Onge MP \& Faith MS (2009) Increased Sweetened Beverage Intake Is Associated with Reduced Milk and Calcium Intake in 3-to 7-Year-Old Children at Multi-Item Laboratory Lunches. J Am Diet Assoc 109(3), 497-501.

2. Bleich SN, Wang YC, Wang Y \& Gortmaker SL (2009) Increasing consumption of sugar-sweetened beverages among US adults: 1988-1994 to 1999-2004. Am J Clin Nutr 89(1), 372-381.

3. Gibson S (2008) Sugar-sweetened soft drinks and obesity: a systematic review of the evidence from observational studies and interventions. Nutrition Research Reviews 21(2), 134-147.

4. Reid M, Hammersley R, Hill AJ \& Skidmore P (2007) Long-term dietary compensation for added sugar: effects of supplementary sucrose drinks over a 4-week period. British Journal of Nutrition 97, 193-203.

5. Reid M, Hammersley R \& Duffy M (2010) Effects of sucrose drinks on macronutrient intake, body weight, and mood state in overweight women over 4 weeks. Appetite 55(1), 130-136. 